Article

\title{
Some Characterizations for a Quaternion-Valued and Dual Variable Curve
}

\author{
Muge Karadag * and Ali Ihsan Sivridag
}

Department of Mathematics, Faculty of Arts and Science, Inonu University, Malatya 44069, Turkey; ali.sivridag@inonu.edu.tr

* Correspondence: muge.karadag@inonu.edu.tr

Received: 23 December 2018; Accepted: 14 January 2019; Published: 22 January 2019

\begin{abstract}
Quaternions, which are found in many fields, have been studied for a long time. The interest in dual quaternions has also increased after real quaternions. Nagaraj and Bharathi developed the basic theories of these studies. The Serret-Frenet Formulae for dual quaternion-valued functions of one real variable are derived. In this paper, by making use of the results of some previous studies, helixes and harmonic curvature concepts in $Q_{\mathbb{D}^{3}}$ and $Q_{\mathbb{D}^{4}}$ are considered and a characterization for a dual harmonic curve to be a helix is given.
\end{abstract}

Keywords: dual quaternion; Helixes; harmonic curvature

MSC: 53A04

\section{Introduction}

First, some fundamental definitions and concepts related to the algebra of dual quaternions are given. A real quaternion $q$ has the form

$$
q=d+a \vec{e}_{1}+b \vec{e}_{2}+c \vec{e}_{3} .
$$

Let $q$ and $q^{*}$ be two real quaternions. A dual quaternion is defined as

$$
\bar{q}=q+\epsilon q^{*}, \varepsilon^{2}=0 .
$$

If

$$
q=d+a \vec{e}_{1}+b \vec{e}_{2}+c \vec{e}_{3} \text { and } q^{*}=d^{*}+a^{*} \vec{e}_{1}+b^{*} \vec{e}_{2}+c^{*} \vec{e}_{3},
$$

then we can write

$$
\bar{q}=D+A \vec{e}_{1}+B \vec{e}_{2}+C \vec{e}_{3}
$$

where $D=d+\varepsilon d^{*}, A=a+\varepsilon a^{*}, B=b+\varepsilon b^{*}$ and $C=c+\varepsilon c^{*}$ are dual numbers. The dual numbers $D$, $A, B$ and $C$ are called dual components of $\bar{q}$ [1].

Hence, a quaternion $\bar{q}$ consists of two parts: the scalar part $S_{\bar{q}}=D$ and the vector part $\vec{V}_{\bar{q}}=A \vec{e}_{1}+$ $B \vec{e}_{2}+C \vec{e}_{3}$. That is, $\bar{q}=S_{\bar{q}}+\vec{V}_{\bar{q}}$, where $S_{\bar{q}}$ is a dual number and $\vec{V}_{\bar{q}}$ is a dual vector [1]. From now on, we show the set of dual quaternions by $Q_{\mathbb{D}^{4}}$.

The sum of two dual quaternions $\bar{p}$ and $\bar{q}$ is defined as

$$
\bar{p}+\bar{q}=(p+q)+\varepsilon\left(p^{*}+q^{*}\right),
$$

where $\bar{p}=p+\varepsilon p^{*}$ and $\bar{q}=q+\varepsilon q^{*}[1]$. 
The product of two dual quaternions $\bar{p}$ and $\bar{q}$ is defined as

$$
\bar{p} \times \bar{q}=p \times q+\varepsilon\left(p \times q^{*}+p^{*} \times q\right),
$$

where the operation $x$ on the right hand side is the real quaternion multiplication, that is,

$$
p \times q=S_{p} S_{q}+S_{p} \vec{V}_{q}+S_{q} \vec{V}_{p}+\vec{V}_{p} \wedge \vec{V}_{q}-<\vec{V}_{p}, \vec{V}_{q}>.
$$

The conjugation of a dual quaternion $\bar{q}=q+\varepsilon q^{*}$ is shown by $\alpha \bar{q}$ and defined by $\alpha \bar{q}=S_{\bar{q}}-\vec{V}_{\bar{q}}$, [1] Let $\mathbb{D}$ be the set of dual numbers. The symmetric $\mathbb{D}$ valued bilinear form $\tilde{h}$ is defined by

$$
\tilde{h}(\bar{p}, \bar{q})=h(p, q)+\varepsilon\left[h\left(p^{*}, q\right)+h\left(p, q^{*}\right)\right]
$$

where $h$ is the inner product on real quaternions defined by

$$
h(p, q)=\frac{1}{2}(p \times \alpha q+q \times \alpha p),
$$

for all real quaternions $p$ and $q[2,3]$.

The norm of a dual quaternion $\bar{p}$ is defined by

$$
\|\bar{p}\|^{2}=\tilde{h}(\bar{p}, \bar{p})=\bar{p} \times \alpha \bar{p} \quad \text { or } \quad\|\bar{p}\|^{2}=D^{2}+A^{2}+B^{2}+C^{2},
$$

where $\|\bar{p}\|^{2}$ is a dual number. If we show the real part by $\operatorname{Re}\|\bar{p}\|^{2}$ and dual part by $D u\|\bar{p}\|^{2}$, then these are:

$$
\operatorname{Re}\|\bar{p}\|^{2}=d^{2}+a^{2}+b^{2}+c^{2}, D u\|\bar{p}\|^{2}=2\left(d d^{*}+a a^{*}+b b^{*}+c c^{*}\right) .
$$

If the norm of a dual quaternion is unit, that is the norm of the real part is one and the norm of the dual part is zero, then this is called unit dual quaternion and is shown as $\bar{p}_{0}$. Furthermore, it can be expressed as

$$
\bar{p}_{0}=D_{0}+A_{0} \vec{e}_{1}+B_{0} \vec{e}_{2}+C_{0} \vec{e}_{3}
$$

where

$$
\begin{gathered}
d_{0}^{2}+a_{0}^{2}+b_{0}^{2}+c_{0}^{2}=1, \\
d_{0} d_{0}^{*}+a_{0} a_{0}^{*}+b_{0} b_{0}^{*}+c_{0} c_{0}^{*}=0 .
\end{gathered}
$$

If $\bar{p}+\alpha \bar{p}=0$, then $\bar{p}$ is called a dual spatial-quaternion. A dual spatial-quaternion may be considered as a dual vector in $\mathbb{D}^{3}$. Dual quaternions $\bar{p}$ and $\bar{q}$ are called $\tilde{h}$-ortogonally if and only if $\tilde{h}(\bar{p}, \bar{q})=0$.

Let $\bar{p}$ and $\bar{q}$ be two unit dual spatial-quaternions. If $\bar{p}$ and $\bar{q}$ are unit dual vectors, then we have

$$
\tilde{h}(\bar{p}, \bar{q})=\cos \phi, \quad \phi=\psi+\varepsilon \psi^{*},
$$

where $\phi$ is the dual angle between $\bar{p}$ and $\bar{q}$ quaternions [1].

In this study, a dual quaternion valued function of a single real variable is called a dual quaternionic curve. Let I be an open interval in $\mathbb{R}$, then a dual quaternionic curve in $Q_{\mathbb{D}^{4}}$ is in the form

$$
\tilde{\beta}(s)=\gamma_{4}(s)+\gamma_{1}(s) \overrightarrow{e_{1}}+\gamma_{2}(s) \overrightarrow{e_{2}}+\gamma_{3}(s) \overrightarrow{e_{3}}+\varepsilon\left[\gamma_{4}^{*}(s)+\gamma_{1}^{*}(s) \overrightarrow{e_{1}}+\gamma_{2}^{*}(s) \overrightarrow{e_{2}}+\gamma_{3}^{*}(s) \overrightarrow{e_{3}}\right]
$$

Throughout the work, we assume that all curves are given with arc-length parameter. Let

$$
\beta(s)=\gamma_{1}(s) \overrightarrow{e_{1}}+\gamma_{2}(s) \overrightarrow{e_{2}}+\gamma_{3}(s) \overrightarrow{e_{3}}+\varepsilon\left[\gamma_{1}^{*}(s) \overrightarrow{e_{1}}+\gamma_{2}^{*}(s) \overrightarrow{e_{2}}+\gamma_{3}^{*}(s) \overrightarrow{e_{3}}\right]
$$


be a spatial-quaternionic curve parameterized by arc-length. With $\left\{T, N_{1}, N_{2}\right\}$ being the Frenet frame field along $\beta$, the Serret-Frenet Formulae of dual spatial-quaternionic curve $\beta$ is given by

$$
\left[\begin{array}{c}
\dot{T} \\
\dot{N}_{1} \\
\dot{N}_{2}
\end{array}\right]=\left[\begin{array}{ccc}
0 & K & 0 \\
-K & 0 & R \\
0 & -R & 0
\end{array}\right]\left[\begin{array}{c}
T \\
N_{1} \\
N_{2}
\end{array}\right]
$$

and $\left(T, N_{1}, N_{2}, R, K\right)$ is the Frenet Apparatus of the curve $\beta$ in $Q_{\mathbb{D}^{3}}$, where $T=t+\varepsilon t^{*}, N_{1}=n_{1}+\varepsilon n_{1}^{*}$, $N_{2}=n_{2}+\varepsilon n_{2}^{*}, K=k+\varepsilon k^{*}$ and $R=r+\varepsilon r^{*}$ are the principal curvature and torsion of $\beta$, respectively. Moreover, $k$ and $r$ are the principal curvature and torsion of the curve in $\mathbb{R}^{3}$, which are determined by the real part of $\beta$, respectively [4].

Theorem 1. Let the quaternionic curve

$$
\tilde{\beta}(s)=D(s)+A(s) \vec{e}_{1}+B(s) \vec{e}_{2}+C(s) \vec{e}_{3}
$$

be derived from the dual spatial-quaternionic curve

$$
\beta(s)=A(s) \vec{e}_{1}+B(s) \vec{e}_{2}+C(s) \vec{e}_{3} .
$$

Then, the Serret-Frenet Formulas for the curve $\tilde{\beta}$ in $Q_{\mathbb{D}^{4}}$ can be derived in the terms of help of the Serret-Frenet vectors of $\beta$ so we have

$$
\left[\begin{array}{c}
\dot{T}^{T} \\
\dot{\tilde{N}}_{1} \\
\dot{\tilde{N}}_{2} \\
\dot{\tilde{N}}_{3}
\end{array}\right]=\left[\begin{array}{cccc}
0 & \tilde{K} & 0 & 0 \\
-\tilde{K} & 0 & K & 0 \\
0 & -K & 0 & R-\tilde{K} \\
0 & 0 & -(R-\tilde{K}) & 0
\end{array}\right]\left[\begin{array}{c}
\tilde{T} \\
\tilde{N}_{1} \\
\tilde{N}_{2} \\
\tilde{N}_{3}
\end{array}\right],
$$

where $\left(\tilde{T}, \tilde{N}_{1}, \tilde{N}_{2}, \tilde{N}_{3}, \tilde{K}, K, R-\tilde{K}\right)$ is the Frenet Apparatus for the curve $\tilde{\beta}$ such that $K$ and $R$ are principal curvature and torsion of the dual spatial-quaternionic curve $\beta$, respectively [5].

\section{Dual Spatial-Quaternionic Helixes and Harmonic Curvatures}

Definition 1. Let $\beta: I \mapsto Q_{\mathbb{D}^{3}}$ be a spatial-quaternionic curve that is parameterized by arc-length $s$ and $u$ be a constant unit dual vector in $Q_{\mathbb{D}^{3}}$. If

$$
\tilde{h}(\dot{\beta}(s), u)=\cos \phi=\cos \left(\varphi+\varepsilon \varphi^{*}\right)=\text { constant, for each } s \in I \quad \varphi \neq \frac{\pi}{2},
$$

then $\beta$ is called a dual spatial-quaternionic helix in $Q_{\mathbb{D}^{3}}$. Let $u=u_{0}+\varepsilon u_{0}^{*}$ be a constant unit dual vector in $Q_{\mathbb{D}^{3}},\{T, N, B\}$ be the Frenet frame field along the spatial-quaternionic curve

$$
\beta(s)=\gamma_{1}(s) \overrightarrow{e_{1}}+\gamma_{2}(s) \overrightarrow{e_{2}}+\gamma_{3}(s) \overrightarrow{e_{3}}+\varepsilon\left[\gamma_{1}^{*}(s) \overrightarrow{e_{1}}+\gamma_{2}^{*}(s) \overrightarrow{e_{2}}+\gamma_{3}^{*}(s) \overrightarrow{e_{3}}\right],
$$

and $\dot{\beta}(s)=T(s), T(s)=T_{0}(s)+\varepsilon T_{0}^{*}(s)$. In addition, the conjugations of $T$ and $u=u_{0}+\varepsilon u_{0}^{*} d u a l$ spatial-quaternions are $\alpha T(s)=\alpha T_{0}(s)+\varepsilon \alpha T_{0}^{*}(s)$ and $\alpha u=-u_{0}-\varepsilon u_{0}^{*}$, respectively.

Thus, from Equation (3), we get

$$
\tilde{h}(T(s), u)=h\left(T_{0}(s), u_{0}\right)+\varepsilon\left[h\left(T_{0}^{*}(s), u_{0}\right)+h\left(T_{0}(s), u_{0}^{*}\right)\right],
$$


where $h$ represents the inner-product on real quaternions. From Equations (3) and (4), we obtain

$$
\begin{aligned}
\tilde{h}(T(s), u) & =\cos \varphi \pm \varepsilon \varphi^{*} \sin \varphi \\
& =\cos \phi .
\end{aligned}
$$

Corollary 1. Let

$$
\gamma(s)=\gamma_{1}(s) \overrightarrow{e_{1}}+\gamma_{2}(s) \overrightarrow{e_{2}}+\gamma_{3}(s) \overrightarrow{e_{3}}
$$

be a helix in $\mathbb{R}^{3}$ and $u$ be a constant unit spatial-quaternion. If $\varphi^{*}=$ constant, then the dual spatial-quaternionic curve $\beta$ given by

$$
\beta(s)=\gamma_{1}(s) \overrightarrow{e_{1}}+\gamma_{2}(s) \overrightarrow{e_{2}}+\gamma_{3}(s) \overrightarrow{e_{3}}+\varepsilon\left[\gamma_{1}^{*}(s) \overrightarrow{e_{1}}+\gamma_{2}^{*}(s) \overrightarrow{e_{2}}+\gamma_{3}^{*}(s) \overrightarrow{e_{3}}\right]
$$

is also a dual spatial-quaternionic helix, where $\varphi^{*}$ is the distance between the lines correspond the dual vectors $T(s)$ and $u$ [3].

Definition 2. Let $\beta: I \mapsto Q_{\mathbb{D}^{3}}$ be a regular dual spatial-quaternionic curve with arc-length parameter $s$ and $T(s), N_{1}(s)$ and $N_{2}(s)$ be the Frenet vectors of $\beta$ at $\beta(s)$. With $u$ being a constant unit dual spatial-quaternion, the function $\tilde{H}$ given by

$$
\tilde{H}: I \mapsto I D, \quad \tilde{h}\left(N_{2}(s), u\right)=\tilde{H}(s) \cos \phi
$$

is called harmonic curvature function of $\beta$ and the dual number $\tilde{H}(s)$ is called the harmonic curvature at $\beta(s)$ with respect to $u$, where $\phi$ is the dual angle between the dual vectors $u=u_{0}+\varepsilon u_{0}^{*}$ and $T(s)$, [3].

Now, we can give the theorem that gives the harmonic curvature in terms of the curvatures of $\beta$.

Theorem 2. Let $\beta: I \mapsto Q_{\mathbb{D}^{3}}$ be a dual spatial-quaternionic helix with arc-length parameter $s$. The harmonic curvature $\tilde{H}(\mathrm{~s})$ of $\beta$ can be given by

$$
\tilde{H}(s)=\frac{K(s)}{R(s)},
$$

where $K$ and $R$ the principal curvature and the torsion of $\beta$, respectively.

Proof. Let $T, N_{1}$ and $N_{2}$ be the Frenet vector fields of the spatial-quaternionic helix $\beta$ and $u$ be a constant unit dual vector. With $\phi$ being the dual angle between $T(s)$ and $u$, we have

$$
\tilde{h}(T(s), u)=\cos \phi=\text { constant }, \quad \phi=\varphi+\varepsilon \varphi^{*} .
$$

By differentiating from the last equation, we get

$$
\tilde{h}(\dot{T}(s), u)=0 .
$$

From Equations (1) and (8), we obtain

$$
\tilde{h}\left(N_{1}(s), u\right)=0 .
$$

By differentiating from Equation (9), we get

$$
\tilde{h}\left(\dot{N}_{1}(s), u\right)=0 .
$$

Taking account of Equations (1) in (10), we obtain

$$
-K(s) \tilde{h}(T(s), u)+R(s) \tilde{h}\left(N_{2}(s), u\right)=0 .
$$


Using Equations (4) and (5) in Equation (11), we get

$$
\tilde{H}(s)=\frac{K(s)}{R(s)} .
$$

Thus, the proof is completed.

Theorem 3. Let $\beta: I \mapsto Q_{\mathbb{D}^{3}}$ be a dual spatial-quaternionic helix with arc-length parameter $s$. If the harmonic curvature at the point $\beta(s)$ is $\tilde{H}(s)$ and $\left\{T(s), N_{1}(s), N_{2}(s)\right\}$ is Frenet frame field for this curve, then $\beta$ is a dual space-quaternionic helix if and only if $\tilde{H}^{2}(s)=$ constant.

Proof. $(\Rightarrow)$ If $\beta$ is a dual space-quaternionic helix, then there is a unit constant $u$ dual vector satisfying the equality

$$
\tilde{h}(\dot{\beta}(s), u)=\cos \phi=\text { constant }, \forall s \in I .
$$

This dual vector expressed in the terms of $T(s), N_{1}(s), N_{2}(s)$ bases of dual space quaternionic $\beta$ curve at the point $\beta(s)$ is given by

$$
u=\tilde{h}(T(s), u) T(s)+\sum_{i=1}^{2} \tilde{h}\left(N_{i}(s), u\right) N_{i}(s) .
$$

If the considered $\|T(s)\|=\left\|N_{1}(s)\right\|=\left\|N_{2}(s)\right\|=1,\|u\|=1$, with the use of Equations (4) and (5), we obtain,

$$
\begin{aligned}
\|u\|^{2} & =\tilde{h}(u, u)=u \times \alpha u \\
1 & =\left[\tilde{h}(T(s), u) T(s)+\sum_{i=1}^{2} \tilde{h}\left(N_{i}(s), u\right) N_{i}(s)\right] \times \alpha\left[\tilde{h}(T(s), u) T(s)+\sum_{i=1}^{2} \tilde{h}\left(N_{i}(s), u\right) N_{i}(s)\right] \\
1 & =\cos ^{2} \phi\|T(s)\|^{2}+\tilde{H}(s) \cos ^{2} \phi T(s) \times \alpha N_{2}(s) \\
& +\tilde{H}(s) \cos ^{2} \phi N_{2}(s) \times \alpha T(s)+\tilde{H}(s) \cos ^{2} \phi\left\|N_{2}(s)\right\|^{2}
\end{aligned}
$$

Since $\|T(s)\|=\left\|N_{2}(s)\right\|=1, T(s) \times \alpha N_{2}(s)=-N_{1}(s)$ and $N_{2}(s) \times \alpha T(s)=N_{1}(s)$, we obtain

$$
\begin{aligned}
\tilde{H}^{2}(s) \cos ^{2} \phi & =1-\cos ^{2} \phi \\
\tilde{H}^{2}(s) & =\frac{\sin ^{2} \phi}{\cos ^{2} \phi}=\tan ^{2} \phi=\text { constant. }
\end{aligned}
$$

$(\Leftarrow)$ Conversely, let us consider $\tilde{H}^{2}(s)=a$ (constant) for $\beta$ dual space-quaternionic curve. In this case, there is a $\phi$ angle that satisfies $\tan ^{2} \phi=a$. Then, we define a dual space quaternion $u$ as

$$
u=\cos \phi T(s)+\tilde{H}(s) N_{2}(s) \cos \phi .
$$

According to this equality,

(1) Dual space-quaternionic $u$ is constant.

(2) $u$ is unit.

(1) We show that dual space $u$ is constant: By taking derivative of Equation (15) with respect to $s$, we obtain

$$
\frac{1}{\cos \phi} \frac{d u}{d s}=\dot{T}(s)+\dot{H}(s) N_{2}(s)+\tilde{H}(s) \dot{N}_{2}(s) .
$$

Here, if we consider the equalities $\dot{T}(s)=K(s) N_{1}(s), \dot{N}_{2}(s)=-R(s) N_{1}(s)$ and $\dot{\tilde{H}}(s)=0$ from $\tilde{H}(s)=\frac{K(s)}{R(s)}$, then we obtain, 


$$
\frac{1}{\cos \phi} \frac{d u}{d s}=K(s) N_{1}(s)+\dot{H}(s) N_{2}(s)+\tilde{H}(s)\left(-R(s) N_{2}(s)\right)=0 .
$$

Furthermore, $u$ is a constant dual space-quaternion.

(2) We show that dual space-quaternion $u$ is unit:

$$
\|u\|^{2}=\tilde{h}(u, u)=u \times \alpha u=\cos ^{2} \phi\left(1+\tan ^{2} \phi\right)=1 .
$$

Thus, $\|u\|=1$. Moreover, we obtain

$$
\begin{aligned}
\tilde{h}(T(s), u) & =\frac{1}{2}[T(s) \times \alpha u+u \times \alpha T(s)] \\
& =\frac{1}{2}\left[\cos \phi\|T(s)\|^{2}+\tilde{H}(s) \cos \phi T(s) \times \alpha N_{2}(s)\right. \\
& \left.+\cos \phi\|T(s)\|^{2}+\tilde{H}(s) \cos \phi N_{2}(s) \times \alpha T(s)\right]=\cos \phi=\text { constant }
\end{aligned}
$$

Thus, it is shown that $\beta$ is a dual space-quaternionic helix.

Here, $\widetilde{T}=\widetilde{t}+\varepsilon \widetilde{t}^{*}, \widetilde{N}_{1}=\widetilde{n}_{1}+\varepsilon \widetilde{n}_{1}^{*}, \widetilde{N}_{2}=\widetilde{n}_{2}+\varepsilon \widetilde{n}_{2}^{*}, \widetilde{N}_{3}=\widetilde{n}_{3}+\varepsilon \widetilde{n}_{3}^{*}, \widetilde{K}=\widetilde{k}+\varepsilon \widetilde{k}^{*}, K=k+\varepsilon k^{*}$, $R-\widetilde{K}=\left(\widetilde{r}+\varepsilon \widetilde{r}^{*}\right)-\left(\widetilde{k}+\varepsilon \widetilde{k}^{*}\right)$.

\section{Dual Quaternionic Helixes in $Q_{\mathbb{D}^{4}}$ and The Harmonic Curvatures of Them}

Definition 3. Let $\tilde{\beta}: I \mapsto Q_{\mathbb{D}^{4}}$ be a dual quaternionic curve such that the tangent vector $\tilde{T}$ of $\tilde{\beta}$ has unit length along $\tilde{\beta}$ and $u$ be a constant unit dual spatial-quaternion. If

$$
\tilde{h}(\dot{\tilde{\beta}}(s), u)=\cos \phi=\text { constant }, \text { for } \forall s \in I,
$$

then $\tilde{\beta}$ is called a dual quaternionic helix [3].

Theorem 4. Let $\tilde{\beta}: I \mapsto Q_{I D^{3}}$ be a dual space quaternionic helix. On the condition,

$$
\beta(s)=A(s) \vec{e}_{1}+B(s) \vec{e}_{2}+C(s) \vec{e}_{3} ; A(s), B(s), C(s) \in I D,
$$

each dual quaternionic helix derived from $\beta$,

$$
\tilde{\beta}(s)=A(s) \vec{e}_{1}+B(s) \vec{e}_{2}+C(s) \vec{e}_{3}+D(s) ; D(s) \in I D
$$

is also a dual quaternionic helix with the same axis of $\beta$ [2].

Definition 4. Let $\tilde{\beta}: I \mapsto Q_{I D^{4}}$ be a regular dual quaternionic curve parameterized by arc-length $s$ and $u$ be constant unit dual spatial-quaternion. Let $\left\{T(s), N_{1}(s), N_{2}(s), N_{3}(s)\right\}$ be the Frenet frame field along $\tilde{\beta}$. With $\phi=\phi(s)$ being the angle between $T(s)$ and $u$, the function $\tilde{H}_{i}: I \mapsto I D,(i=1,2)$, defined by

$$
\tilde{h}\left(\tilde{N}_{i+1}(s), u\right)=\tilde{H}_{i}(s) \cos \phi
$$

is called the harmonic curvature function of $\tilde{\beta}$, of order $i$. We define also $\tilde{H}_{0}=0$.

We can give the theorem as follows:

Theorem 5. Let $\tilde{\beta}: I \mapsto Q_{\mathbb{D}^{4}}$ be a dual quaternionic curve with the arc-length parameter $s$. Then, there are the following relations between the curvatures and harmonic curvatures:

$$
\tilde{H}_{1}(s)=\frac{\tilde{K}(s)}{K(s)} \quad \text { and } \quad \tilde{H}_{2}(s)=\frac{\dot{\tilde{H}}_{1}(s)}{(R-\tilde{K})(s)} .
$$


Proof. Let $u$ be a constant unit dual spatial-quaternion and $\tilde{T}(s), \tilde{N}_{1}(s), \tilde{N}_{2}(s)$ and $\tilde{N}_{3}(s)$ be the Frenet vectors of $\tilde{\beta}$ at the point $\tilde{\beta}(s)$. Since $\tilde{\beta}(s)$ is a helix, we have

$$
\tilde{h}(\tilde{T}(s), u)=\cos \phi=\text { constant }
$$

By differentiating from the last equation, we obtain that

$$
\tilde{h}(\dot{\widetilde{T}}(s), u)=0 .
$$

From Equation (2), $\tilde{H}_{0}=0$ and Equation (20), we obtain $\tilde{h}\left(\tilde{N}_{1}(s), u\right)=0$. By differentiating the last equation, we have

$$
\tilde{h}\left(\dot{\tilde{N}}_{1}(s), u\right)=0 .
$$

By using Equation (2) in Equation (21), we obtain

$$
-\tilde{K}(s) \tilde{h}(\tilde{T}(s), u)+K(s) \tilde{h}\left(\tilde{N}_{2}(s), u\right)=0 .
$$

Since

$$
\tilde{h}\left(\tilde{N}_{2}(s), u\right)=\tilde{H}_{1}(s) \cos \phi,
$$

from Equation (22) and the last equation, we obtain

$$
\tilde{H}_{1}(s)=\frac{\tilde{K}(s)}{K(s)}
$$

where $\tilde{K}(s)$ and $K(s)$ are the first and second curvatures of $\tilde{\beta}$, respectively. By differentiating Equation (23), we get

$$
\tilde{h}\left(\dot{\tilde{N}}_{2}(s), u\right)=\dot{\tilde{H}}_{1}(s) \cos \phi .
$$

From Equations (2) and (24), we obtain

$$
-K(s) \tilde{h}\left(\tilde{N}_{1}(s), u\right)+(R-\tilde{K})(s) \tilde{h}\left(\tilde{N}_{3}(s), u\right)=\dot{\tilde{H}}_{1} \cos \phi .
$$

Considering Equations (19) from (25), we get

$$
\tilde{H}_{2}(s)=\frac{\dot{\tilde{H}}_{1}(s)}{(R-\tilde{K})(s)}
$$

or

$$
\tilde{H}_{2}(s)=\dot{\tilde{H}}_{1}(s) \tilde{\sigma_{3}}(s) .
$$

Thus, the proof is completed.

Theorem 6. Let $\tilde{\beta}: I \mapsto Q_{\mathbb{D}^{4}}$ be a dual quaternionic helix with arc-length parameter $s$, $\left\{\tilde{T}(s), \tilde{N}_{1}(s), \tilde{N}_{2}(s), \tilde{N}_{3}(s)\right\}$ be Frenet frame field for this curve at the point $\tilde{\beta}(s)$ and $\tilde{H}_{i}(s), i=1,2$ be the harmonic curvature. Then, $\tilde{\beta}$ is a quaternionic helix $\Leftrightarrow \sum_{i=1}^{2} \tilde{H}_{i}^{2}(s)=$ constant.

Proof. $(\Rightarrow)$ Let us consider $\tilde{\beta}: I \mapsto Q_{\mathbb{D}^{4}}$ is a helix. Then, there is a unit and constant dual space-quaternion $u$ for curve $\tilde{\beta}$, which satisfies

$$
\tilde{h}(\dot{\tilde{\beta}}(s), u)=\cos \phi=\text { constant, } \forall s \in I .
$$


This dual space quaternion is expressed as

$$
u=\tilde{h}(\tilde{T}(s), u) \tilde{T}(s)+\sum_{i=1}^{3} \tilde{h}\left(\tilde{N}_{i}(s), u\right) \tilde{N}_{i}(s)
$$

in terms of the base $\tilde{\beta}(s)$. From Equations (18) and (19), since $\tilde{T}(s), \tilde{N}_{1}(s), \tilde{N}_{2}(s), \tilde{N}_{3}(s)$ and $u$ are units, we obtain

$$
\begin{aligned}
\|u\|^{2} & =\tilde{h}(u, u)=u \times \alpha u \\
1 & =\left[\tilde{h}(\tilde{T}(s), u) \tilde{T}(s)+\sum_{i=1}^{3} \tilde{h}\left(\tilde{N}_{i}(s), u\right) \tilde{N}_{i}(s)\right] \times \alpha[\tilde{h}(\tilde{T}(s), u) \tilde{T}(s) \\
& \left.+\sum_{i=1}^{3} \tilde{h}\left(\tilde{N}_{i}(s), u\right) \tilde{N}_{i}(s)\right] .
\end{aligned}
$$

Hence,

$$
\sum_{i=1}^{2} \tilde{H}_{i}^{2}(s)=\tan ^{2} \phi=\text { constant. }
$$

$(\Leftarrow)$ : Let us receive $\sum_{i=1}^{2} \tilde{H}_{i}^{2}(s)=\tan ^{2} \phi=a$ (constant) for $\tilde{\beta}: I \mapsto Q_{\mathbb{D}^{4}}$ dual quaternionic curve. In this case, there is a dual angle $\phi$ satisfying $\tan ^{2} \phi=a$. According to this, the dual space quaternion defined as

$$
u=\cos \phi \tilde{T}(s)+\sum_{i=2}^{3} \tilde{H}_{i-1}(s) \cos \phi \tilde{N}_{i}(s)
$$

is a constant and unit quaternion.

(1) Let us once again show $u$ is constant: By taking derivative of Equation (30) with respect to $s$, we obtain

$$
\frac{1}{\cos \phi} \frac{d u}{d s}=\dot{\tilde{T}}(s)+\sum_{i=2}^{3} \dot{\tilde{H}}_{i-1}(s) \tilde{N}_{i}(s)+\sum_{i=2}^{3} \tilde{H}_{i-1}(s) \dot{\tilde{N}}_{i}(s) .
$$

On the other hand, if we rewrite for $i=2$ Equation (19),

$$
\tilde{h}\left(\tilde{N}_{3}(s), u\right)=\tilde{H}_{2}(s) \cos \phi
$$

and we re-derive with respect to $s$, we obtain

$$
\begin{gathered}
\left.\tilde{h}_{\left(\dot{\tilde{N}}_{3}\right.}(s), u\right)=\dot{\tilde{H}}_{2}(s) \cos \phi \\
\dot{\tilde{H}}_{2}(s)=-(R-\tilde{K})(s) \tilde{H}_{1}(s) .
\end{gathered}
$$

By using Equations (2) and (24) in Equation (31) with $\dot{\tilde{H}}_{1}(s)=(R-\tilde{K})(s) \tilde{H}_{2}(s)$,

$$
\begin{aligned}
\frac{1}{\cos \phi} \frac{d u}{d s} & =\tilde{K}(s) \tilde{N}_{1}(s)+(R-\tilde{K})(s) \tilde{H}_{2}(s) \tilde{N}_{2}(s)-(R-\tilde{K})(s) \tilde{H}_{1}(s) \tilde{N}_{3}(s) \\
& +\frac{\tilde{K}(s)}{K(s)}\left(-\tilde{K}(s) \tilde{N}_{1}(s)+(R-\tilde{K})(s) \tilde{N}_{3}(s)\right. \\
& +\frac{\tilde{H}_{1}(s)}{(R-\tilde{K})(s)}\left(-(R-\tilde{K})(s) \tilde{N}_{2}(s)\right) \frac{1}{\cos \phi} \frac{d u}{d s} \\
& =0 .
\end{aligned}
$$

Hence, $u$ is constant space quaternion. 
(2) Let us show $u$ is unit: We can write,

$$
\begin{aligned}
\|u\|^{2} & =\tilde{h}(u, u)=u \times \alpha u \\
& =\left[\cos \phi \tilde{T}(s)+\sum_{i=2}^{3} \tilde{H}_{i-1}(s) \cos \phi \tilde{N}_{i}(s)\right] \times\left[\cos \phi \tilde{T}(s)+\sum_{i=2}^{3} \tilde{H}_{i-1}(s) \cos \phi \tilde{N}_{i}(s)\right]=1
\end{aligned}
$$

thus, $\|u\|=1$.

On the other hand, using the definition of $u$, we can find

$$
\begin{aligned}
& \tilde{h}(\tilde{T}(s), u)=\frac{1}{2}[\tilde{T}(s) \times \alpha u+u \times \alpha \tilde{T}(s)] \\
& \tilde{h}(\tilde{T}(s), u)=\cos \phi=\text { constant. }
\end{aligned}
$$

Thus, we obtain $\tilde{\beta}$ is a helix and hence the proof is completed.

Corollary 2. The equations of derivative of the harmonic curvatures given by Equations (26) and (33) for dual quaternionic curve in matrix form are expressed as

$$
\left[\begin{array}{c}
\dot{H}_{1} \\
\dot{H}_{2}
\end{array}\right]=\left[\begin{array}{cc}
0 & (R-\tilde{K}) \\
-(R-\tilde{K}) & 0
\end{array}\right]\left[\begin{array}{l}
\tilde{H}_{1} \\
\tilde{H}_{2}
\end{array}\right] .
$$

Author Contributions: All authors contributed significantly to the content of the paper. M.K. wrote the manuscript and the co-authors reviewed it.

Funding: This research received o external funding.

Conflicts of Interest: The authors declare no competing interests.

\section{References}

1. Bharathi, K.; Nagaraj, M. Geometry of Quaternionic and Pseudo-Quaternionic Multiplications. Ind. J. Pure Appl. Math. 1985, 16, 741-756.

2. Bharathi, K.; Nagaraj, M. Quaternion Valued Function of a Real Variable Serret-Frenet Formulae. Ind. J. Pure Appl. Math. 1987, 18, 507-511.

3. Hacisalihoğlu, H.H. Motion Geometry and Quaternions Theory; Gazi University Faculty of Arts and Science Publications: Ankara, Turkey, 1983.

4. Karadağ, M. Helixes and Characterisations on the Quaternionic Lorentz Manifolds. Ph.D. Thesis, Inonu University, Malatya, Turkey, 1999.

5. Sivridağ, A.I.; Güneş, R.; Keleş, S. The Serret-Frenet Formulae for Dual Quaternion Valued Functions of a single real Variable. Mech. Mach. Theory 1994, 29, 749-754. [CrossRef]

(C) 2019 by the authors. Licensee MDPI, Basel, Switzerland. This article is an open access article distributed under the terms and conditions of the Creative Commons Attribution (CC BY) license (http://creativecommons.org/licenses/by/4.0/). 